\title{
2. Dokumentation
}

Otmar Jung

a) Internationaler Bereich (Auswahl)

Litauen, 14. Oktober 2012

Referendum über den Bau eines neuen Atomkraftwerks

Frage: „I support the construction of a new nuclear power plant in the Republic of Lithuania“"

\begin{tabular}{|l|l|l|l|l|l|l|l|l|}
\hline $\begin{array}{l}\text { Stimm- } \\
\text { berechtigte }\end{array}$ & $\begin{array}{l}\text { Abgegebene } \\
\text { Stimmen }\end{array}$ & in \% & ungültig & gültig & Ja & in \% & Nein & in \% \\
\hline $\mathbf{2 . 5 8 8 . 4 1 8}$ & 1.361 .082 & 52,6 & 43.953 & 1.317 .129 & 463.966 & 35,2 & 853.163 & 64,8 \\
\hline
\end{tabular}

Ergebnis: Der Bau eines neuen Atomkraftwerks wurde abgelehnt.

Nach: The Central Electoral Commission of the Republic of Lithuania: Election to the Seimas and the Referendum on the Construction of a New Nuclear Power Plant in the Republic of Lithuania, Voting Results (18.10.2012), http://www.vrk.lt/2012 seimo_rinki-mai/output_en/referendumas/referendumas.html (Zugriff 5.1.2013).

Island, 20. Oktober 2012

Konsultatives Parlamentsplebiszit über einzelne Themen der geplanten Verfassung

„Do you wish the Constitution Council's proposals to form the basis of a new draft Constitution?"

\begin{tabular}{|c|l|r|r|r|r|r|r|r|}
\hline $\begin{array}{l}\text { Stimm- } \\
\text { berechtigte }\end{array}$ & $\begin{array}{l}\text { Abgegebene } \\
\text { Stimmen }\end{array}$ & in \% & ungültig & gültig & Ja & in \% & Nein & in \% \\
\hline $\mathbf{2 3 6 . 8 5 0}$ & 115.890 & 48,9 & 4.759 & 109.811 & 73.509 & 66,9 & 36.302 & 33,1 \\
\hline
\end{tabular}

Ergebnis: Die Mehrheit der Abstimmenden folgte der Empfehlung der Verfassungskommission.

„In the new Constitution, do you want natural resources that are not privately owned to be declared national property?“ 


\begin{tabular}{|r|l|l|l|l|l|l|l|l|}
\hline $\begin{array}{l}\text { Stimm- } \\
\text { berechtigte }\end{array}$ & $\begin{array}{l}\text { Abgegebene } \\
\text { Stimmen }\end{array}$ & in \% & ungültig & gültig & Ja & in \% & Nein & in \% \\
\hline $\mathbf{2 3 6 . 8 5 0}$ & 115.890 & 48,9 & 12.340 & 102.230 & 84.760 & 82,9 & 17.470 & 17,1 \\
\hline
\end{tabular}

Ergebnis: Die Mehrheit der Abstimmenden folgte der Empfehlung der Verfassungskommission.

„Would you like to see provisions in the new Constitution on an established (national) church in Iceland?“

\begin{tabular}{|c|l|l|l|l|l|l|l|l|}
\hline $\begin{array}{l}\text { Stimm- } \\
\text { berechtigte }\end{array}$ & $\begin{array}{l}\text { Abgegebene } \\
\text { Stimmen }\end{array}$ & in \% & ungültig & gültig & Ja & in \% & Nein & in \% \\
\hline $\mathbf{2 3 6 . 8 5 0}$ & 115.890 & 48,9 & 12.201 & 102.369 & 58.455 & 57,1 & 43.914 & 42,9 \\
\hline
\end{tabular}

Ergebnis: Die Mehrheit der Abstimmenden folgte nicht der Empfehlung der Verfassungskommission; diese hatte keine Bestimmungen über eine Staatskirche in Island vorgesehen.

„Would you like to see a provision in the new Constitution authorising the election of particular individuals to the Althingi more than is the case at present? “

\begin{tabular}{|r|l|l|l|l|l|l|l|l|}
\hline $\begin{array}{l}\text { Stimm- } \\
\text { berechtigte }\end{array}$ & $\begin{array}{l}\text { Abgegebene } \\
\text { Stimmen }\end{array}$ & in \% & ungültig & gültig & Ja & in \% & Nein & in \% \\
\hline $\mathbf{2 3 6 . 8 5 0}$ & 115.890 & 48,9 & 14.459 & 100.111 & 78.451 & 78,4 & 21.660 & 21,6 \\
\hline
\end{tabular}

Ergebnis: Die Mehrheit der Abstimmenden folgte der Empfehlung der Verfassungskommission.

„Would you like to see a provision in the new Constitution giving equal weight to votes cast in all parts of the country?"

\begin{tabular}{|c|l|l|l|l|l|l|l|l|}
\hline $\begin{array}{l}\text { Stimm- } \\
\text { berechtigte }\end{array}$ & $\begin{array}{l}\text { Abgegebene } \\
\text { Stimmen }\end{array}$ & in \% & ungültig & gültig & Ja & in \% & Nein & in \% \\
\hline $\mathbf{2 3 6 . 8 5 0}$ & 115.890 & 48,9 & 14.327 & 100.243 & 66.653 & 66,5 & 33.590 & 33,5 \\
\hline
\end{tabular}

Ergebnis: Die Mehrheit der Abstimmenden folgte der Empfehlung der Verfassungskommission.

„Would you like to see a provision in the new Constitution stating that a certain proportion of the electorate is able to demand that issues are put to a referendum?"“ 


\begin{tabular}{|r|l|l|l|l|l|l|l|l|}
\hline $\begin{array}{l}\text { Stimm- } \\
\text { berechtigte }\end{array}$ & $\begin{array}{l}\text { Abgegebene } \\
\text { Stimmen }\end{array}$ & in \% & ungültig & gültig & Ja & in \% & Nein & in \% \\
\hline $\mathbf{2 3 6 . 8 5 0}$ & 115.890 & 48,9 & 15.497 & 99.073 & 72.633 & 73,3 & 26.440 & 26,7 \\
\hline
\end{tabular}

Ergebnis: Die Mehrheit der Abstimmenden folgte der Empfehlung der Verfassungskommission.

Nach: http://www.statice.is/temp_en/Dialog/Print.asp?Matrix=KOS04108 \&timeid=2013-424125851\&lang=1; $\mathrm{http}: / / \mathrm{www}$.statice.is $/$ ?PageID=2465 $\&$ src $=/$ temp_en/Dialog/vaval.-asp?ma $=$ KOS04105\%26ti $=$ Votes + cast $\% 2 C+$ voters+and+participation+by+municipality + in + the + referendum $+2010-2012$ $+++\% 26$ path $=$./Database/kosningar/thjodaratkv/\%26lang=1\%26units=Number/percent (Zugriff 24.4.2013). Zahl der gültigen Stimmen und Prozentsatz der Ja- bzw. Nein-Stimmen: eigene Berechnung.

Irland, 10. November 2012

\section{Children's Referendum}

Frage: „Do you approve of the proposal to amend the Constitution contained in the undermentioned Bill?" [Thirty-first Amendment of the Constitution (Children) Bill 2012]

\begin{tabular}{|c|l|l|l|l|l|l|l|r|}
\hline $\begin{array}{l}\text { Stimm- } \\
\text { berechtigte }\end{array}$ & $\begin{array}{l}\text { Abgegebene } \\
\text { Stimmen }\end{array}$ & in \% & ungültig & gültig & Ja & in \% & Nein & in \% \\
\hline $\mathbf{3 . 1 8 3 . 6 8 6}$ & 1.066 .239 & 33,5 & 4.645 & 1.061 .594 & 615.731 & 58 & 445.863 & 42 \\
\hline
\end{tabular}

Nach: http://www.referendum.ie/results.php (Zugriff 12.2.2013). - Ergebnis: Die Verfassungsänderung wurde angenommen.

Österreich, 20. Januar 2013

\section{Volksbefragung zur Wehrpflicht}

Lösungsvorschlag a) Sind Sie für die Einführung eines Berufsheeres und eines bezahlten freiwilligen Sozialjahres?

Lösungsvorschlag b) Sind Sie für die Beibehaltung der allgemeinen Wehrpflicht und des Zivildienstes?

\begin{tabular}{|l|l|l|l|l|l|l|l|l|}
\hline $\begin{array}{l}\text { Stimm- } \\
\text { berechtigte }\end{array}$ & $\begin{array}{l}\text { Abgegebene } \\
\text { Stimmen }\end{array}$ & in \% & ungültig & gültig & $\begin{array}{l}\text { Lösungs- } \\
\text { vorschlag } \\
\text { a) }\end{array}$ & in \% & $\begin{array}{l}\text { Lösungs- } \\
\text { vorschlag } \\
\text { b) }\end{array}$ & $\begin{array}{l}\text { in } \\
\%\end{array}$ \\
\hline $\mathbf{6 . 3 7 8 . 6 2 8}$ & 3.344 .620 & 52,4 & 82.226 & 3.262 .394 & 1.315 .278 & 40,3 & 1.947 .116 & 59,7 \\
\hline
\end{tabular}


Nach: Bundesministerium für Inneres: Endergebnis inkl. Briefwahlstimmen, nach: http://vb2013.bmi.gv.at/ (Zugriff 13.2.2013). - Ergebnis: Die Mehrheit stimmte für die Beibehaltung der allgemeinen Wehrpflicht.

Bulgarien, 27. Januar 2013

\section{Referendum über den Bau eines neuen Atomkraftwerks}

Frage: „Soll die Kernenergie in der Republik Bulgarien durch den Bau eines neuen Kernreaktors gefördert werden?“

\begin{tabular}{|c|l|l|l|l|l|l|l|l|}
\hline $\begin{array}{l}\text { Stimm- } \\
\text { berechtigte }\end{array}$ & $\begin{array}{l}\text { Abgegebene } \\
\text { Stimmen }\end{array}$ & in \% & ungültig & gültig & Ja & in \% & Nein & in \% \\
\hline $\mathbf{6 . 9 5 2 . 1 8 3}$ & 1.405 .463 & 20,2 & 20.180 & 1.385 .283 & 851.757 & 61,5 & 533.526 & 38,5 \\
\hline
\end{tabular}

Nach:

http://c2d.ch/detailed_display.php? 1 name $=$ votes\&table $=$ votes\&page $=1 \&$ parent $i d=\&$ sublinkname $=$ results\&id $=132760$ (Zugriff 14.3.2013). Hinweis: Nach diesen Angaben ist Zahl der abgegebenen Stimmen gleich der Zahl der gültigen Stimmen, und diese liegt höher als die Summe der Ja- und NeinStimmen (1.385.283); daher abgeklärt nach: http://referendum.cik.bg/ ?resh=148 (Zugriff 14.3.2013). - Ergebnis: Der Bau eines neuen Atomkraftwerks wurde mehrheitlich befürwortet. Da jedoch das Beteiligungsquorum verfehlt wurde (Beteiligung mindestens so hoch wie bei der letzten Parlamentswahl), ist das Ergebnis nicht bindend.

Falklandinseln, 10. und 11. März 2013

\section{Referendum über den politischen Status}

Frage: „Do you wish the Falkland Islands to retain their current political status as an Overseas Territory of the United Kingdom?"

\begin{tabular}{|r|r|r|r|r|r|r|r|r|}
\hline $\begin{array}{l}\text { Stimm- } \\
\text { berechtigte }\end{array}$ & $\begin{array}{l}\text { Abgegebene } \\
\text { Stimmen }\end{array}$ & in \% & ungültig & \multicolumn{1}{l|}{ gültig } & Ja & in \% & Nein & in \% \\
\hline $\mathbf{1 . 6 5 0}$ & 1.518 & 92,0 & 1 & 1.517 & 1.513 & 99,8 & 3 & 0,2 \\
\hline
\end{tabular}

Nach: http://www.falklands.gov.fk/results-of-the-referendum-on-the-political-status-of-the-falkland-islands/ (Zugriff 23.4.2013). Absolute Zahl der Stimmberechtigten: eigene Berechnung. „1 vote was unaccounted for.“ - Ergebnis: Die überwältigende Mehrheit votierte für die Beibehaltung des jetzigen politischen Status. 
Frankreich, Départements Bas-Rhin und Haut-Rhin, 7. April 2013

Referendum über die Zusammenlegung der Administrationen der beiden Départements und der Région zu einem Einheitsrat für das Elsaß

Frage: „Approuvez-vous le projet de création, en Alsace, d'une Collectivité territoriale d'Alsace, par fusion du Conseil régional d'Alsace, du Conseil général du Bas-Rhin et du Conseil général du Haut-Rhin?”

Département Bas-Rhin

\begin{tabular}{|c|l|l|l|l|l|l|l|l|}
\hline $\begin{array}{l}\text { Stimm- } \\
\text { berechtigte }\end{array}$ & $\begin{array}{l}\text { Abgegebene } \\
\text { Stimmen }\end{array}$ & in \% & ungültig & gültig & Ja & in \% & Nein & in \% \\
\hline $\mathbf{7 5 1 . 5 2 7}$ & 263.837 & 35,11 & 8.923 & 254.914 & 172.137 & 67,53 & 82.777 & 32,47 \\
\hline
\end{tabular}

Département Haut-Rhin

\begin{tabular}{|r|l|l|l|l|l|l|l|l|}
\hline $\begin{array}{l}\text { Stimm- } \\
\text { berechtigte }\end{array}$ & $\begin{array}{l}\text { Abgegebene } \\
\text { Stimmen }\end{array}$ & in \% & ungültig & gültig & Ja & in \% & Nein & in \% \\
\hline $\mathbf{5 2 1 . 3 2 1}$ & 193.848 & 37,18 & 5.736 & 188.112 & 83.266 & 44,26 & 104.846 & 55,74 \\
\hline
\end{tabular}

Total Région Alsace

\begin{tabular}{|c|l|l|l|l|l|l|l|l|}
\hline $\begin{array}{l}\text { Stimm- } \\
\text { berechtigte }\end{array}$ & $\begin{array}{l}\text { Abgegebene } \\
\text { Stimmen }\end{array}$ & in \% & ungültig & gültig & Ja & in \% & Nein & in \% \\
\hline $\mathbf{1 . 2 7 2 . 8 4 8}$ & 457.685 & 35,96 & 14.659 & 443.026 & 255.403 & 57,65 & 187.623 & 42,35 \\
\hline
\end{tabular}

Nach: http://www.interieur.gouv.fr/Elections/Les-resultats/Locales/Referendum-du-7-avril-2013-concernant-1-Alsace (Zugriff 13.8.2013). Voraussetzung für einen Erfolg waren eine Mehrheit der Abstimmenden in beiden Départements sowie die Erfüllung eines jeweils 25-prozentigen Zustimmungsquorums. - Ergebnis: Das Projekt scheiterte an der Nein-Mehrheit im Département Haut-Rhin.

Irland, 4. Oktober 2013

\section{Abolition of Seanad Éireann}

Frage: „Do you approve of the proposal to amend the Constitution contained in the undermentioned Bill?" [Thirty-second Amendment of the Constitution (Abolition of Seanad Éireann) Bill 2013] 


\begin{tabular}{|l|l|l|l|l|l|l|l|l|}
\hline $\begin{array}{l}\text { Stimm- } \\
\text { berechtigte }\end{array}$ & $\begin{array}{l}\text { Abgegebene } \\
\text { Stimmen }\end{array}$ & in \% & ungültig & gültig & Ja & in \% & Nein & in \% \\
\hline $\mathbf{3 . 1 6 7 . 3 8 4}$ & 1.240 .729 & 39,17 & 14.355 & 1.226 .374 & 591.937 & 48,27 & 634.437 & 51,73 \\
\hline
\end{tabular}

Nach: http://www.referendum.ie/results.php?ref=9 (Zugriff 20.11.2013). - Ergebnis: Die Verfassungsänderung wurde abgelehnt.

Irland, 4. Oktober 2013

\section{Court of Appeal}

Frage: „Do you approve of the proposal to amend the Constitution contained in the undermentioned Bill?" [Thirty-third Amendment of the Constitution (Court of Appeal) Bill 2013]

\begin{tabular}{|l|l|l|l|l|l|l|l|l|}
\hline $\begin{array}{l}\text { Stimm- } \\
\text { berechtigte }\end{array}$ & $\begin{array}{l}\text { Abgegebene } \\
\text { Stimmen }\end{array}$ & in \% & ungültig & gültig & Ja & in \% & Nein & in \% \\
\hline $\mathbf{3 . 1 6 7 . 3 8 4}$ & 1.240 .135 & 39,15 & 20.080 & 1.220 .055 & 795.008 & 65,16 & 425.047 & 34,84 \\
\hline
\end{tabular}

Nach: http://www.referendum.ie/results.php?ref=8 (Zugriff 20.11.2013). - Ergebnis: Die Verfassungsänderung wurde angenommen.

Kroatien, 1. Dezember 2013

Referendum über die Verankerung der Ehe als einer Gemeinschaft zwischen Mann und Frau in der Verfassung

Frage: „Sind Sie dafür, dass die Bestimmung, wonach die Ehe eine Gemeinschaft zwischen Mann und Frau ist, in die Verfassung der Republik Kroatien eingetragen wird?" (nach: http://www.wienerzeitung.at/nachrichten/europa/europastaaten/591158_Mit-direkter-Demokratie-gegen-Minderheiten-Rechte.html [Zugriff 5.12.2013]).

\begin{tabular}{|l|l|l|l|l|l|l|l|l|}
\hline $\begin{array}{l}\text { Stimm- } \\
\text { berechtigte }\end{array}$ & $\begin{array}{l}\text { Abgegebene } \\
\text { Stimmen }\end{array}$ & in \% & ungültig & gültig & Ja & in \% & Nein & in \% \\
\hline $\mathbf{3 . 7 9 1 . 0 0 0}$ & 1.436 .163 & 37,88 & 8.196 & 1.427 .967 & 946.433 & 66,3 & 481.534 & 33,7 \\
\hline
\end{tabular}

Nach: http://www.izbori.hr/2013Referendum/rezult/r_00_0000_000.html?t= 138-7790266477 (Zugriff 23.12.2013). Prozentwerte für „Ja“ und „Nein“ eigene Berechnung, da die amtlichen Veröffentlichungen auf die abgegebenen, anstatt auf die abgegebenen gültigen Stimmen prozentuieren. - Ergebnis: Die Referendumsfrage ist bejaht. 
b) Deutschland

aa) Daten zu Volksbegehren und Volksentscheid

Bayern, 17. bis 30. Januar 2013

Volksbegehren „, Grundrecht auf Bildung ernst nehmen - Studienbeiträge abschaffen!“

Am 12. November 2012 machte das Bayerische Staatsministerium des Innern den diesem Volksbegehren zugrunde liegenden Gesetzentwurf bekannt, vgl. Bay-StAnz. Nr. 46 v. 16.11.2012.

\begin{tabular}{|c|c|c|c|}
\hline Stimmberechtigte & Gültige Eintragungen & in $\%$ & Quorum in \% \\
\hline 9.438 .854 & 1.352 .618 & 14,3 & 10 \\
\hline
\end{tabular}

Bek. des Landeswahlleiters des Endgültigen Ergebnisses v. 20. Februar 2013, BayStAnz. Nr. 8 v. 22.2.2013. Kleinräumige Aufgliederung unter http://www.wahlen.bayern.de/vb-ve/index.php (Zugriff 15.3.2013), ferner unter Volksbegehren in Bayern 2013. Statistischer Bericht Kennziffer B VII 4/1-4 2013. - Ergebnis: Das Volksbegehren ist zustande gekommen.

Berlin, 11. Februar bis 10. Juni 2013

Volksbegehren „über die Rekommunalisierung der Berliner Energieversorgung“"

Am 17. Januar 2013 machte die Landesabstimmungsleiterin den diesem Volksbegehren zugrundliegenden Gesetzentwurf bekannt, vgl. ABl. S. 110 (Nr. 4 v. 25.1.2013).

\begin{tabular}{|r|r|r|r|}
\hline Stimmberechtigte & Gültige Eintragungen & \multicolumn{1}{|c|}{ in \% } & \multicolumn{1}{|c|}{ Quorum } \\
\hline $\mathbf{2 . 4 8 3 . 6 3 9}$ & 227.748 & 9,2 & 7 \\
\hline
\end{tabular}

Bek. der Landesabstimmungsleiterin des Endgültigen Ergebnisses v. 25. Juni 2013, ABl. S. 1282 (Nr. 29 v. 5.7.2013). Prozentangabe nach der Pressemitteilung der Landesabstimmungsleiterin v. 25. Juni 2013, https://www.wahlen-berlin.de/Abstimmungen/VB2013_NEnergie/presse/201 30625.pdf? sel1 $=5059 \&$ sel2 $=1500$ (Zugriff 13.8.2013). Dort auch Aufgliederung nach Bezirken. - Ergebnis: Das Volksbegehren ist zustande gekommen. 
Bayern, 15. September 2013

Volksentscheide über den Beschluss des Bayerischen Landtags vom 20. Juni 2013

LT-Drs. 16/17358, Bekanntmachung der Bayerischen Staatsregierung vom 2. Juli 2013 Az.: B II 2 - G 4/91, http://www.wahlen.bayern.de/volksentscheide/2013-bek_staatsregierung-020713R.pdf (Zugriff 13.8.2013).

A. Gesetz zur Änderung der Verfassung des Freistaates Bayern - „Förderung gleichwertiger Lebensverhältnisse und Arbeitsbedingungen“"

\begin{tabular}{|c|l|l|l|l|l|l|l|l|}
\hline $\begin{array}{l}\text { Stimmbe- } \\
\text { rechtigte }\end{array}$ & $\begin{array}{l}\text { Abgegebene } \\
\text { Stimmen }\end{array}$ & in \% & ungültig & gültig & Ja & in \% & Nein & in \% \\
\hline $\mathbf{9 . 4 4 1 . 1 2 5}$ & 5.962 .063 & 63,1 & 452.603 & 5.509 .460 & 4.936 .357 & 89,6 & 573.103 & 10,4 \\
\hline
\end{tabular}

B. Gesetz zur Änderung der Verfassung des Freistaates Bayern - „Förderung des ehrenamtlichen Einsatzes für das Gemeinwohl““

\begin{tabular}{|l|l|l|l|l|l|l|l|r|}
\hline $\begin{array}{l}\text { Stimm- } \\
\text { berechtigte }\end{array}$ & $\begin{array}{l}\text { Abgegebene } \\
\text { Stimmen }\end{array}$ & in \% & ungültig & gültig & Ja & in \% & Nein & in \% \\
\hline $\mathbf{9 . 4 4 1 . 1 2 5}$ & 5.962 .063 & 63,1 & 564.019 & 5.398 .044 & 4.894 .924 & 90,7 & 503.120 & 9,3 \\
\hline
\end{tabular}

C. Gesetz zur Änderung der Verfassung des Freistaates Bayern - „Angelegenheiten der Europäischen Union“"

\begin{tabular}{|c|l|l|l|l|l|l|l|l|}
\hline $\begin{array}{l}\text { Stimm- } \\
\text { berechtigte }\end{array}$ & $\begin{array}{l}\text { Abgegebene } \\
\text { Stimmen }\end{array}$ & in \% & ungültig & gültig & Ja & in \% & Nein & in \% \\
\hline $\mathbf{9 . 4 4 1 . 1 2 5}$ & 5.962 .063 & 63,1 & 710.003 & 5.252 .060 & 4.418 .721 & 84,1 & 833.339 & 15,9 \\
\hline
\end{tabular}

D. Gesetz zur Änderung der Verfassung des Freistaates Bayern - „Schuldenbremse“"

\begin{tabular}{|c|l|l|l|l|l|l|l|l|}
\hline $\begin{array}{l}\text { Stimm- } \\
\text { berechtigte }\end{array}$ & $\begin{array}{l}\text { Abgegebene } \\
\text { Stimmen }\end{array}$ & in \% & ungültig & gültig & Ja & in \% & Nein & in \% \\
\hline $\mathbf{9 . 4 4 1 . 1 2 5}$ & 5.962 .063 & 63,1 & 616.094 & 5.345 .969 & 4.738 .907 & 88,6 & 607.062 & 11,4 \\
\hline
\end{tabular}

E. Gesetz zur Änderung der Verfassung des Freistaates Bayern - „Angemessene Finanzausstattung der Gemeinden"

\begin{tabular}{|c|l|l|l|l|l|l|l|r|}
\hline $\begin{array}{l}\text { Stimm- } \\
\text { berechtigte }\end{array}$ & $\begin{array}{l}\text { Abgegebene } \\
\text { Stimmen }\end{array}$ & in \% & ungültig & gültig & Ja & in \% & Nein & in \% \\
\hline $\mathbf{9 . 4 4 1 . 1 2 5}$ & 5.962 .063 & 63,1 & 610.763 & 5.351 .300 & 4.902 .018 & 91,6 & 449.282 & 8,4 \\
\hline
\end{tabular}


Absolute Zahlen nach Bek. des Landeswahlleiters des Freistaates Bayern vom 16.10.2013: Ergebnisse der Volksentscheide zur Änderung der Verfassung des Feistaates Bayern am 15. September 2013, in: BayStAnz. Nr. 43 v. 25.10.2013, S. 5.

Aufgliederung nach Regierungsbezirken, Kreisen und Gemeinden unter http://www.volksentscheide2013.bayern.de (Zugriff 26.11.2013) sowie unter: Volksentscheide zur Änderung der Verfassung des Freistaates Bayern am 15. September 2013. Endgültiges Ergebnis, hrsg. vom Bayerischen Landesamt für Statistik und Datenverarbeitung, München 2013 (Statistischer Bericht Kennziffer B VII 4/2-2 2013). Dort auch die Prozentwerte für Beteiligung, „Ja“ und „Nein“. - Ergebnis: Die zur Abstimmung gestellten Gesetzentwürfe wurden durch die Volksentscheide angenommen.

Die entsprechenden fünf Gesetze zur Änderung der Verfassung des Freistaates Bayern, jeweils vom 11.11.2013, wurden verkündet in GVB1. S. 638, $639,640.641$ und 642.

Hamburg, 22. September 2013

\section{Volksentscheid ,Unser Hamburg - Unser Netz“}

„Senat und Bürgerschaft unternehmen fristgerecht alle notwendigen und zulässigen Schritte, um die Hamburger Strom-, Fernwärme- und Gasleitungsnetze 2015 wieder vollständig in die Öffentliche Hand zu übernehmen. Verbindliches Ziel ist eine sozial gerechte, klimaverträgliche und demokratisch kontrollierte Energieversorgung aus erneuerbaren Energien."

Stimmen Sie dieser Vorlage zu?

\begin{tabular}{|c|l|l|l|l|l|l|l|l|}
\hline $\begin{array}{l}\text { Stimm- } \\
\text { berechtigte }\end{array}$ & $\begin{array}{l}\text { Abgegebene } \\
\text { Stimmen }\end{array}$ & in \% & ungültig & gültig & Ja & in \% & Nein & in \% \\
\hline $\mathbf{1 . 2 9 3 . 1 0 2}$ & 888.300 & 68,7 & 14.968 & 873.332 & 444.352 & 50,9 & 428.980 & 49,1 \\
\hline
\end{tabular}

Siehe das endgültige Ergebnis des Volksentscheids unter http://www.hamburg.de/wahlen/4125972/ergebnis-volksentscheid-energienetze.html, Aufgliederung nach den Hamburger Stadtteilen (ohne Briefwahl) unter http://www.statistik-nord.de/uploads/tx_standocuments/Stadtteile_Volksentscheid_Energienetze_.pdf (Zugriff jeweils 20.11.2013).

Zum speziellen Hamburger Quorum: Die Anzahl der Ja-Stimmen muss größer sein als die Hälfte der Zweitstimmen, die bei der Bundestagswahl in Hamburg auf die Parteien abgegeben wurden, die mindestens ein Bundestagsmandat erhalten haben (vgl. Art. 50 Abs. 3 Satz 10 Verfassung). Die Anzahl von $444.352 \mathrm{Ja}$-Stimmen entspricht im Umfang der Mehrheit der in dem 
gleichzeitig gewählten Bundestag repräsentierten Hamburger Stimmen (382.976). Nach dem vom Bundeswahlausschuss am 9. Oktober 2013 festgestellten amtlichen Endergebnis der Bundestagswahl sind im 18. Deutschen Bundestag die Parteien CDU, CSU, SPD, DIE LINKE und GRÜNE vertreten. Diese Parteien haben in Hamburg insgesamt 765.951 Zweitstimmen erhalten. Die Mehrheit beträgt damit 382.976 Stimmen. - Ergebnis: Die Vorlage der Volksinitiative „Unser Hamburg - Unser Netz“ ist angenommen.

Brandenburg, 10. April bis 9. Oktober 2013

\section{Volksbegehren „Hochschulen erhalten“}

Am 28. Januar 2013 machte der Landesabstimmungsleiter den Wortlaut dieses Volksbegehrens bekannt, ABl. S. 423 (Nr. 7 v. 20.2.2013).

\begin{tabular}{|r|r|r|r|}
\hline Stimmberechtigte & Gültige Eintragungen & in \% & \multicolumn{1}{l|}{ Quorum in \% } \\
\hline $\mathbf{2 . 0 9 1 . 4 6 0}$ & 18.105 & 0,9 & $80.000=3,83$ \\
\hline
\end{tabular}

Zahl der gültigen Eintragungen nach der Bek. des Gesamtergebnisses v. 5.12.2013, GVBl. für das Land Brandenburg Teil I o. S. (Nr. 45). Zahl der Stimmberechtigten und Prozentsatz der Eintragungen nach der PM 18/2013 des Landesabstimmungsleiters v. 24.10.2013. Prozentwert des Quorums: eigene Berechnung. - Ergebnis: Das Volksbegehren ist nicht zustande gekommen.

Berlin, 3. November 2013

Volksentscheid „,über die Rekommunalisierung der Berliner Energieversorgung"

Abstimmungsfrage auf dem Stimmzettel:

Abgestimmt wird über den Entwurf des Gesetzes „Gesetz für die demokratische, ökologische und soziale Energieversorgung in Berlin (Energie VG)“, der im Amtsblatt für Berlin vom 20. September 2013 veröffentlicht ist und im Wesentlichen folgende Zielsetzung hat:

- Errichtung von Stadtwerken als Anstalt öffentlichen Rechts, um Strom aus erneuerbaren Energien zu erzeugen und Energieeinsparmöglichkeiten zu nutzen

- Errichtung einer Netzgesellschaft als Anstalt öffentlichen Rechts mit dem Ziel, die Stromnetze zum 1.1.2015 zu übernehmen 
- Entgegenwirken von Energiearmut (Energiearmut ist der mangelnde Zugang zu bezahlbaren Energiedienstleistungen)

- Schaffung von demokratischen Beteiligungsmöglichkeiten durch Direktwahl des Verwaltungsrats, Initiativrecht und Versammlungen

Stimmen Sie diesem Gesetzentwurf zu?

\begin{tabular}{|c|l|l|l|l|l|l|l|l|}
\hline $\begin{array}{l}\text { Stimm- } \\
\text { berechtigte }\end{array}$ & $\begin{array}{l}\text { Abgegebene } \\
\text { Stimmen }\end{array}$ & in \% & ungültig & gültig & Ja & in \% & Nein & in \% \\
\hline $\mathbf{2 . 4 8 3 . 7 5 6}$ & 722.109 & 29,1 & 1.408 & 720.701 & 599.588 & 83,2 & 121,113 & 16,8 \\
\hline
\end{tabular}

Absolute Zahlen (außer ,gültig“) nach Bek. des Endgültigen Ergebnisses v. 14.11.2013, ABl. S. 2399. Vgl. Bericht der Landesabstimmungsleiterin: Volksentscheid über die Rekommunalisierung der Berliner Energieversorgung am 3. November 2013. Endgültiges Ergebnis, zugleich Statistischer Bericht B VII 4-1 (mit Aufgliederung nach Bezirken), https:/www.wahlenberlin.de/historie/Abstimmungen/Landesabstimmungsleiterinbericht_VE13. pdf (Zugriff 22.11.2013). - „Gültige Stimmen“ eigene Berechnung. Prozentwerte für „Ja“ und „Nein“ eigene Berechnung, da die amtlichen Veröffentlichungen auf die abgegebenen, anstatt auf die abgegebenen gültigen Stimmen prozentuieren. - Ergebnis: Da die Zahl von 599.588 Ja-Stimmen $24,1 \%$ der Stimmberechtigten entsprach, war das 25prozentige Zustimmungsquorum nach Art. 63 Abs. 1 Satz 3 Berl.Verf. nicht erreicht und das volksbegehrte Gesetz nicht zustande gekommen.

bb) Daten zu Bürgerbegehren und Bürgerentscheid (Auswahl)

Münster, 16. September 2012

Bürgerentscheid ,Umbenennung des Hindenburgplatzes“

Frage: „Soll der Ratsbeschluss vom 21.3.2012 über die Umbenennung des Hindenburgplatzes aufgehoben werden und damit der Platz den Namen Hindenburgplatz behalten?“

\begin{tabular}{|r|r|r|r|r|r|r|r|r|}
\hline $\begin{array}{l}\text { Stimm- } \\
\text { berechtigte }\end{array}$ & $\begin{array}{l}\text { Abgegebene } \\
\text { Stimmen }\end{array}$ & in \% & ungültig & gültig & Ja & in \% & Nein & in \% \\
\hline $\mathbf{2 3 7 . 7 2 7}$ & 95.827 & 40,3 & 311 & 95.516 & 38.800 & 40,6 & 56.716 & 59,4 \\
\hline
\end{tabular}

Endgültiges Ergebnis nach: Bek. des Abstimmungsleiters v. 19.9.2012, in: Amtsblatt der Stadt Münster, S. 125 (Nr. 18 v. 28.9.2012). Prozentwerte eigene Berechnung. - Ergebnis: Da die Mehrheit, welche die Abstimmungs- 
frage mit „Nein“ beantwortet hatte, mindestens 10 Prozent der Stimmberechtigten (=23.773) betrug, war der Bürgerentscheid gültig.

Aachen, 10. März 2013

Bürgerentscheid „Campusbahn“

Frage: „Sind Sie für den Bau der Campusbahn?“

\begin{tabular}{|r|l|l|l|l|l|l|l|l|}
\hline $\begin{array}{l}\text { Stimm- } \\
\text { berechtigte }\end{array}$ & $\begin{array}{l}\text { Abgegebene } \\
\text { Stimmen }\end{array}$ & in \% & ungültig & gültig & Ja & in \% & Nein & in \% \\
\hline $\mathbf{1 9 2 . 6 3 6}$ & 82.843 & 43,0 & 177 & 82.666 & 27.825 & 33,7 & 54.841 & 66,3 \\
\hline
\end{tabular}

Nach: http://wahlen.regioit-aachen.de/RBE13/05334002/index.htm (Zugriff 15.3.2013). Dort auch kleinräumige Aufgliederung. - Ergebnis: Der Bürgerentscheid ist gültig. Die Aachener Bürger haben sich gegen den Bau der Campus-Bahn entschieden.

Mannheim, 22. September 2013

\section{Bürgerentscheid zur Durchführung der Bundesgartenschau 2023}

Frage: „Soll Mannheim zur nachhaltigen Entwicklung eines Grünzugs Nordost im Jahr 2023 eine Bundesgartenschau durchführen, die überwiegend auf dem Gelände der ehemaligen Spinelli-Kaserne und unter Einbeziehung einer maximal 16 Hektar großen Teilfläche der Feudenheimer Au unter Beibehaltung ihres Status als Landschaftsschutzgebiet stattfindet?“

\begin{tabular}{|r|l|l|l|l|l|l|l|l|}
\hline $\begin{array}{l}\text { Stimm- } \\
\text { berechtigte }\end{array}$ & $\begin{array}{l}\text { Abgegebene } \\
\text { Stimmen }\end{array}$ & in \% & ungültig & gültig & Ja & in \% & Nein & in \% \\
\hline $\mathbf{2 2 9 . 9 9 6}$ & 136.750 & 59,5 & 2.068 & 134.682 & 68.312 & 50,7 & 66.370 & 49,3 \\
\hline
\end{tabular}

Endergebnis nach: Bek. des Fachbereichs Rat, Beteiligung und Wahlen v. 2.10. 2013, ABl. Stadt Mannheim Nr. 115 v. 3.10.2013, S. 4. Prozentwerte eigene Berechnung. - Ergebnis: Da die Zahl der gültigen Ja-Stimmen mehr als 25 Prozent der Stimmberechtigten beträgt, ist ein bindender Bürgerentscheid zustande gekommen. Die Mannheimer Bürger haben sich für die Durchführung der Bundesgartenschau 2023 entschieden.

10. November 2013

Bürgerentscheide zur Bewerbung der Landeshauptstadt München u.a. um die Olympischen und Paralympischen Winterspiele 2022 
Frage: „Sind Sie dafür, dass sich die Landeshauptstadt München zusammen mit der Marktgemeinde Garmisch-Partenkirchen und den Landkreisen Berchtesgadener Land und Traunstein um die Olympischen und Paralympischen Winterspiele 2022 bewirbt?

München

\begin{tabular}{|c|l|l|l|l|l|l|l|l|}
\hline $\begin{array}{l}\text { Stimm- } \\
\text { berechtigte }\end{array}$ & $\begin{array}{l}\text { Abgegebene } \\
\text { Stimmen }\end{array}$ & in \% & ungültig & gültig & Ja & in \% & Nein & in \% \\
\hline $\mathbf{1 . 0 7 3 . 2 2 1}$ & 310.598 & 28,9 & 293 & 310.305 & 148.479 & 47,8 & 161.826 & 52,2 \\
\hline
\end{tabular}

Nach: Bek. des stellv. Abstimmungsleiters v. 20.11.2013, ABl. der Landeshauptstadt München S. 460. „Gültige Stimmen“ sowie Prozentwerte für „Ja“ und „Nein" eigene Berechnung, da die amtlichen Veröffentlichungen auf die abgegebenen, anstatt auf die abgegebenen gültigen Stimmen prozentuieren (der Unterschied wird allerdings bei nur einer Dezimalen nicht sichtbar). Das Abstimmungsquorum von $10 \%$ der Stimmberechtigten wurde erreicht. Der Bürgerentscheid ist gültig. - Ergebnis: Die Münchner Bürger haben sich gegen die Bewerbung um die Olympischen Spiele 2022 entschieden.

Garmisch-Partenkirchen

\begin{tabular}{|r|r|r|r|r|r|r|l|r|}
\hline $\begin{array}{l}\text { Stimm- } \\
\text { berechtigte }\end{array}$ & $\begin{array}{l}\text { Abgegebene } \\
\text { Stimmen }\end{array}$ & in \% & ungültig & gültig & Ja & in \% & Nein & in \% \\
\hline $\mathbf{2 1 . 1 2 7}$ & 11.789 & 55,8 & 28 & 11.761 & 5.696 & 48,4 & 6.065 & 51,6 \\
\hline
\end{tabular}

Nach: Absolute Zahlen nach Bek. des Abstimmungsleiters v. 12.11.2013, freundlicherweise mitgeteilt vom Wahlamt des Markts GarmischPartenkirchen. Prozentzahlen eigene Berechnung. Das Abstimmungsquorum von 20 Prozent der Stimmberechtigten wurde erreicht. Der Bürgerentscheid ist gültig. - Ergebnis: Die Bürger des Markts Garmisch-Partenkirchen haben sich gegen die Bewerbung um die Olympischen Spiele 2022 entschieden.

Landkreis Berchtesgadener Land

\begin{tabular}{|r|l|r|r|r|r|r|l|r|}
\hline $\begin{array}{l}\text { Stimm- } \\
\text { berechtigte }\end{array}$ & $\begin{array}{l}\text { Abgegebene } \\
\text { Stimmen }\end{array}$ & in \% & ungültig & gültig & \multicolumn{1}{l|}{ Ja } & in \% & Nein & in \% \\
\hline $\mathbf{8 3 . 5 7 6}$ & 31.966 & 38,2 & 47 & 31.919 & 14.652 & 45,9 & 17.267 & 54,1 \\
\hline
\end{tabular}

Nach: Bek. des Abstimmungsleiters v. 11.11.2013, ABl. für den Landkreis Berchtesgadener Land usw. Nr. 47 v. 19.11.2013, S. 305. Prozentwerte eigene Berechnung. Das Abstimmungsquorum von zehn Prozent der Stimmberechtigten wurde erreicht. Der Bürgerentscheid ist gültig. - Ergebnis: Die Bürger des Landkreises Berchtesgaden haben sich gegen die Bewerbung um die Olympischen Spiele 2022 entschieden. 
Landkreis Traunstein

\begin{tabular}{|r|l|l|l|l|l|l|l|l|}
\hline $\begin{array}{l}\text { Stimm- } \\
\text { berechtigte }\end{array}$ & $\begin{array}{l}\text { Abgegebene } \\
\text { Stimmen }\end{array}$ & in \% & ungültig & gültig & Ja & in \% & Nein & in \% \\
\hline $\mathbf{1 3 9 . 1 0 6}$ & 55.537 & 39,9 & 63 & 55.474 & 22.374 & 40,3 & 33.100 & 59,7 \\
\hline
\end{tabular}

Nach: Bek. des Abstimmungsleiters v. 12.11.2013, ABl. für den Landkreis Traunstein Nr. 36 v. 15.11.2013, S. 161. Prozentwerte eigene Berechnung. Das Abstimmungsquorum von zehn Prozent der Stimmberechtigten wurde erreicht. Der Bürgerentscheid ist gültig. - Ergebnis: Die Bürger des Landkreises Traunstein haben sich gegen die Bewerbung um die Olympischen Spiele 2022 entschieden. 